\title{
Improving the Patient-Clinician and Parent- Clinician Partnership in Atopic Dermatitis Management
}

\author{
Anthony J. Mancini, MD, ${ }^{*}$ Amy S. Paller, MD, ${ }^{\dagger}$ \\ Eric L. Simpson, MD, MCR, ${ }^{\ddagger}$ Charles N. Ellis, MD, ${ }^{\S}$ and Lawrence F. Eichenfield, MD"
}

\begin{abstract}
Long-term adherence to carefully developed, individualized strategies is necessary for the optimum treatment outcomes in patients with atopic dermatitis (AD). However, the parents of children with $A D$ frequently lack sufficient information about the disease and its treatment, hold incorrect and sometimes harmful beliefs about these issues, and too often do not follow through consistently with the treatment plan. The health care provider is the primary source of such education, so an effective provider relationship is fundamental to adherence. In addition to the provision of correct information and the correction of misinformation, clinicians must be aware of and must address barriers to adherence with AD therapy, especially parent anxiety about the safety of topical medications (corticosteroids and topical calcineurin inhibitors).
\end{abstract}

Semin Cutan Med Surg 31(suppl 3):S23-S28 (C) 2012 Elsevier Inc. All rights reserved.

KEYWORDS atopic dermatitis, steroid phobia, psychiatric comorbidities, treatment adherence

A ggressive topical therapy is one of the most important steps in the treatment of atopic dermatitis (AD). The efficacy of even the most careful and considered treatment regimens is only as good as the parents' or patients' ability and willingness to implement the clinical recommendations.

*Professor of Pediatrics and Dermatology, Northwestern University Feinberg School of Medicine, and Head, Division of Pediatric Dermatology, Ann \& Robert H. Lurie Children's Hospital of Chicago, Chicago, IL.

†Walter J. Hamlin Professor and Chair, Department of Dermatology, Professor of Pediatrics, Northwestern University Feinberg School of Medicine, Attending Physician, Ann \& Robert Lurie Children's Hospital of Chicago, Chicago, IL

₹Associate Professor of Dermatology and Director of Clinical Studies, Oregon Health and Science University, Portland, OR.

§William B. Taylor Professor of Clinical Dermatology, Associate Chair, Department of Dermatology, University of Michigan Medical Center, Ann Arbor, MI.

|Professor of Clinical Pediatrics and Medicine (Dermatology), Chief, Pediatric and Adolescent Dermatology, University of California, San Diego School of Medicine, Rady Children's Hospital, San Diego, CA.

Publication of this CME article was jointly sponsored by the University of Louisville Continuing Health Sciences Education and Global Academy for Medical Education LLC in affiliation with Skin Disease Education Foundation and is supported by an educational grant from Valeant Pharmaceuticals North America Inc.

Lawrence F. Eichenfield, MD has served as a consultant for Anacor, Bayer, and Onset Therapeutics and as a speaker and consultant for Valeant. He has also been an investigator and consultant for Galderma and Leo Pharma as well as an investigator for Amgen, Astellas Pharma US, and Stiefel, A GSK Company.
Adherence is an important issue that must be considered in any clinical encounter in which therapy is a component; with chronic diseases such as $\mathrm{AD}$, which require long-term maintenance and care, low adherence on the part of the patient or parent can be especially problematic. Treatment outcomes can be improved with better understanding of, and improvement in, adherence. ${ }^{1}$ This article addresses the key components of topical treatment that must be implemented and

Charles N. Ellis, MD, has served as a consultant for Galderma, Ferndale Laboratories, Medicis, and Novartis.

Anthony J. Mancini, MD, has served as a consultant for Quinnova and Valeant as well as a speaker and consultant for Galderma.

Amy S. Paller, MD, has received grant research support from Astellas.

Eric L. Simpson, MD, MCR, has served as a consultant, investigator, and speaker for Galderma.

The faculty have received an honorarium from Global Academy for Medical Education for their participation in this activity. They acknowledge the editorial assistance of Joanne Still, medical writer, and Global Academy for Medical Education in the development of this continuing medical education journal article. Joanne Still has no relevant financial relationships with any commercial interests.

Address reprint requests to: Anthony Mancini, MD, Professor of Pediatrics and Dermatology, Northwestern University's Feinberg School of Medicine; Head, Division of Pediatic Dermatology; Director, Pediatric Dermatology Fellowship Program, Ann and Robert H. Lurie Children's Hospital of Chicago, Chicago. 225 East Chicago Avenue, Box 107, Chicago, IL 606112605, Telephone: 312-227-6060; Fax: 312-227-9402. E-mail: amancini@ northwestern.edu 
followed by caregivers or, in the case of older children and adults, by patients themselves.

\section{Obstacles to Adherence}

Adherence requires understanding, understanding requires education, and misinformation and unfounded fears are the main obstacles to both of these requirements. Parents of children with $\mathrm{AD}$ often have preconceived notions about triggers and "allergies" as well as concerns about toxicities of AD treatments.

Despite widespread professional and lay literature describing the evidence-based conclusions about the efficacy and safety of topical anti-inflammatories used in the treatment of $\mathrm{AD}$, steroid phobia (or, as it is often called in the United Kingdom, "corticophobia") and fear of topical calcineurin inhibitors (TCIs), both persist. The results of one recent study summarize the problem succinctly. Aubert-Wastiaux and colleagues $^{2}$ distributed a 69-item questionnaire to 208 consecutive patients with $\mathrm{AD}$ attending the outpatient dermatology clinics of several centers. Completed questionnaires were received from 144 parents and 87 adult patients. Among the responders, $80.7 \%$ reported having fears about topical corticosteroids, and 36\% admitted to treatment nonadherence. The investigators note correlations between steroid phobia and several factors, including the belief that topical corticosteroid agents pass through the skin into the bloodstream, a lack of trust in the practitioner, and discrepancies in the education received about their use.

\section{The Scope of the Nonadherence Problem}

Poor adherence to a prescribed treatment regimen should always be considered in patients in whom the response to therapy is suboptimal. A classic study by Richards and colleagues ${ }^{3}$ showed that medications prescribed for chronic conditions are not taken as prescribed an estimated 30\% to $40 \%$ of the time.

In addition to phobias regarding the use of prescribed treatments (especially topical corticosteroids and TCIs, as described above), underlying reasons for poor adherence among patients and families of children with $\mathrm{AD}$ include family conflicts, parental involvement/caregiver fatigue, and misunderstandings regarding "toxicities" (for instance, the hypopigmentation often noted following inflammatory dermatoses, which parents may mistakenly identify as a toxicity of the topical medication [Figure 1]).

In a study of 37 children with mild to moderate $A D$ treated with triamcinolone ointment twice daily, electronic monitors were used to measure adherence over the 8-week study period. Patients were unaware of the monitoring until they were informed at the conclusion of the study. In the 26 patients who completed the study, mean adherence was $32 \%$. Interestingly_but not surprisingly_adherence was higher on or near office visit days and subsequently decreased rapidly. ${ }^{4}$

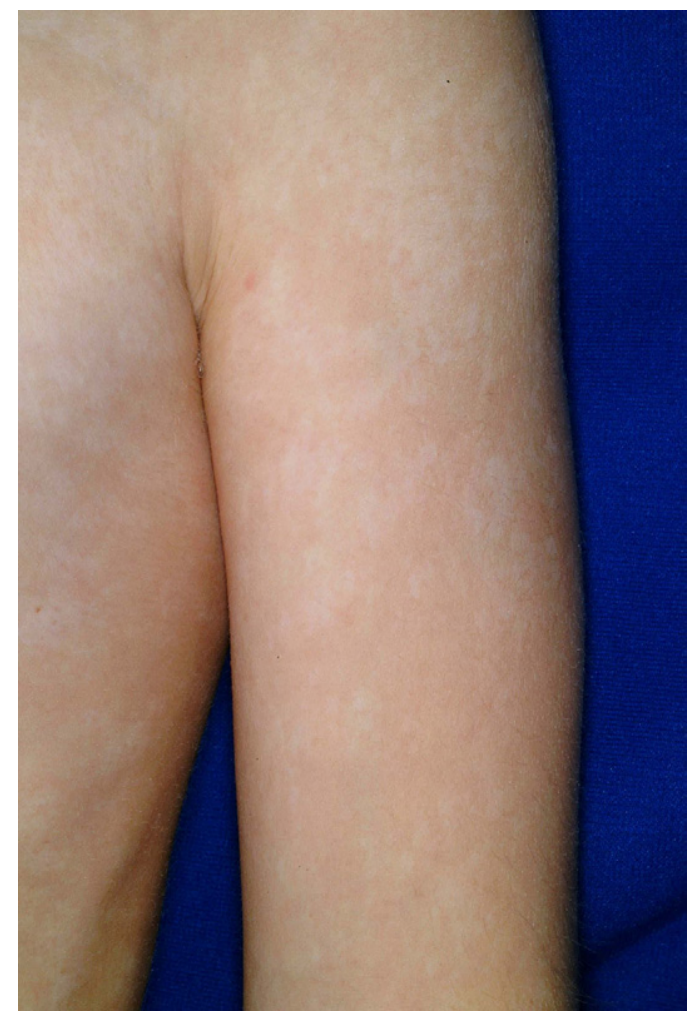

Figure 1 Postinflammatory hypopigmentation in AD. Post-treatment clearing of $\mathrm{AD}$ often reveals hypopigmentation or other skin discoloration. Parents should be reassured that these changes are the result of $\mathrm{AD}$, are not a side effect of topical medication, and will gradually resolve over time without intervention. Photo courtesy of Anthony J. Mancini, MD.

\section{Bathing in Atopic Dermatitis}

Historically, bathing approaches have been divided into socalled dry and wet methods. The preponderance of evidence, as well as widespread clinical experience, supports the superior benefits of the wet method. Nevertheless, some clinicians still consider the dry method to be an acceptable option, and some parents and patients are advised to use it.

The dry method consists of minimization of bathing and is based on the hypothesis that overbathing contributes to exacerbation of skin xerosis. The earliest recommendations for the dry method came from Scholtz, ${ }^{5,6}$ in which bathing or washing was strictly prohibited and the skin was cleansed only with a lipid-free lotion. In 2009, Chiang and colleagues ${ }^{7}$ published the results of a crossover study in five pediatric patients with $\mathrm{AD}$ (and a parallel group with healthy skin) in whom objective parameters of cutaneous hydration status were assessed following various combinations of bathing and moisturizing regimens. The methods were bathing alone without emollient, bathing and immediate emollient application, bathing and delayed emollient application, and emollient application alone. Emollient alone yielded a significantly greater mean hydration over 90 minutes than did bathing with immediate emollient, bathing and delayed emollient, and bathing alone. Emollient application after 


\section{Analogy-The Intuitive Story}

Misinformation can be absorbed intellectually and may be transformed into a firmly held belief. Correcting such beliefs by making statements of fact and discussing evidence works for some individuals, but not for all, and with regard to some information, but not all.

The analogy is a story through which a person can understand a concept in terms of another concept that is intuitively understood and accepted. A simple analogy can be an effective tool for education and re-education, particularly if it is clearly presented and accompanied by demonstrations or illustrations.

The following are two "scripts" that one of the authors (AJM) routinely uses in AD education of parents and patients.

The Diaper Area Analogy: Explaining Why the Wet Method Works

"Atopic dermatitis likes dry skin, but not moist and hydrated skin. Look at the area covered by your baby's diaper. Feel how soft and supple it is? And notice that eczema does not occur in this area. By bathing your baby every day and immediately applying the medications and then the moisturizer, we are trying to create the same sort of environment (a moist one) as in the diaper area on the rest of your baby's skin."

The Footprints in the Snow Analogy: Explaining Why Postinflammatory Hypopigmentation Is Not a Medication Side Effect

"I am walking in the woods on a winter's day, and I see a scary animal in my path. I chase the animal away, and the only thing left are its footprints. The footprints are temporary, and they will disappear with time.

Atopic dermatitis is like the animal in the snow. We have chased it away with the medication I prescribed. As you can see, the eczema ('the animal') is now gone, and the light spots that remain are the 'footprints in the snow.' The medication did not cause them, but rather, the atopic dermatitis did. These 'footprints' will also disappear over time.'

bathing improved hydration, whereas bathing without emollient application yielded poorer hydration status.

The wet method—also referred to as the "soak and smear" technique ${ }^{8}$ — consists of regular daily bathing for short periods of time, followed by the application of topical medications and emollients or barrier repair agents. Hydration for 20 minutes followed by ointment application to wet skin was found to be associated with objective and symptomatic improvement in-and was well accepted by-28 patients referred to a tertiary center for refractory chronic inflammatory dermatoses. ${ }^{8}$

Extensive clinical experience has demonstrated that the wet method is highly useful in the treatment of inflammatory dermatoses, including $\mathrm{AD}$. Bathing provides physical debridement of sloughed cells from, and reduction in superficial microorganisms on, the skin surface, hydration of the stratum corneum, and enhanced penetration of topical agents. It also provides some emotional and social benefits to patients. For example, for infants and toddlers, bath time supports parent-child bonding, particularly when play is incorporated into the event; for older patients, bathing provides a feeling of cleanliness and promotes a sense of wellbeing. The "diaper area analogy" (see "Analogy-The Intuitive Story" above) frequently used by one of the authors (AJM) may help parents understand why the wet method works (Figure 2).

\section{Updating Information, Correcting Misinformation}

\section{Allergen Avoidance}

The nature of $\mathrm{AD}$ and the multifactorial aspects involving genetic predisposition and variability in exacerbating factors make trigger identification very challenging. Potential triggers may include climate change, bacterial colonization, environmental irritants, stress, inhalant allergens, and foods.

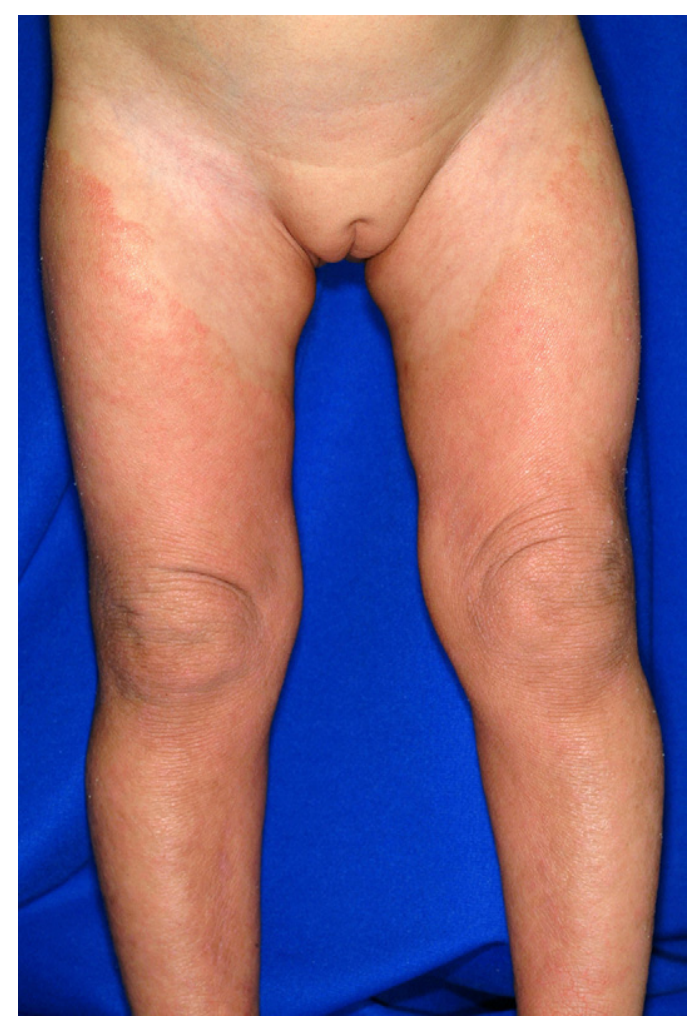

Figure 2 Diaper Area Spared in AD. The look and feel of the skin in the area covered by a child's diaper illustrates the benefit of moisture in mitigating $\mathrm{AD}$-associated dryness and inflammation. Photo courtesy of Anthony J. Mancini, MD. 
Many clinicians and parents have been taught (and still believe) that simple allergen avoidance will significantly alter the burden of disease.

The association between $\mathrm{AD}$ and other atopic disorders (allergic rhinoconjunctivitis, asthma, and food and environmental allergies) makes it challenging for patients and parents to understand the lack of a consistent or predictable direct cause-and-effect relationship.

The most recent evidence demonstrates that allergen avoidance may alter the clinical course in some patients, but it rarely plays as significant a role as once was believed. Although food allergy may result in a variety of skin reactions, it is only the minority of these reactions that truly exacerbate AD. Food allergy should be defined as clinically significant, immunologically-mediated adverse health effects consistently evident with food consumption. In studies of oral food challenges in patients with $\mathrm{AD}$, the majority of food reactions occurred early after food exposure, usually in the form of urticaria. ${ }^{9}, 10$ Delayed reactions, in the form of AD exacerbation, comprise only $5 \%$ to $25 \%$ of oral food challenge reactions and are not predictable by skin-prick or immunoglobulin E serum testing. ${ }^{11}$ Parents should be cautioned against extreme restriction diets, which most often are unhelpful and which may lead to serious nutritional deficits. ${ }^{12,13}$

Recently issued revised Food Allergy Guidelines from the National Institute of Allergy and Infectious Diseases ${ }^{14,15}$ suggest that food allergy evaluation is warranted in children less than 5 years of age under two circumstances: (1) if they have persistent AD despite optimum management or (2) if they have a reliable history of food reactions. The guidelines recommend evaluating such children for allergies to cow's milk, eggs, peanuts, and wheat and soy products. Counseling should include information that food allergy tests (specific IgE and skin prick tests) have poor specificity; predictive values are low, around $20 \%$ to $25 \%$; only one in four to five positive tests correlates with a clinically significant allergy. ${ }^{11}$

\section{Anti-inflammatory Therapy}

Great strides have been made in recent years in describing and understanding the crucial role of skin barrier defects in patients with $\mathrm{AD}$, and a great deal of attention is being paidappropriately so-to the importance of maintaining and restoring skin barrier integrity. However, the role of anti-inflammatory medication should not be overshadowed by these emerging discoveries. In patients with $\mathrm{AD}$, controlling inflammation is still a fundamental goal.

Topical corticosteroids remain the cornerstone of first-line treatment strategies. Clinicians must always emphasize that these medications are safe and effective when prescribed and administered appropriately.

Education also is important regarding the role of TCIs; those currently available in the United States are pimecrolimus and tacrolimus. These agents are approved by the US Food and Drug Administration (FDA) for children 2 years of age or older for second-line treatment of moderate to severe $\mathrm{AD}$. The TCIs also are often used as monotherapy for managing mild to moderate flares and as maintenance therapy between flares. In addition, many clinicians prefer TCIs over topical corticosteroids for treating periorbital $\mathrm{AD}$, given their superior risk/toxicity profile (compared to topical corticosteroids) for treatment of this area. Topical pimecrolimus has been shown to be effective in preventing $\mathrm{AD}$ disease flares and reducing the need for topical corticosteroids in longer-term, intermittent-use trials. Its use has also been associated with improvements in the healthrelated quality of life in pediatric patients with $\mathrm{AD}$ and their parents. ${ }^{16}$

Concerns expressed in the lay media and the presence of a boxed warning in the prescribing information for TCIs have alarmed some practitioners and parents. However, these concerns should be mitigated by the accumulated body of evidence demonstrating the safety of TCIs. The FDA-mandated patient registries for tacrolimus (APPLES) ${ }^{17}$ and pimecrolimus (Pediatric Eczema Elective Registry; PEER) have enrolled more than 12,000 patients to date, and no increased malignancy signal above the Surveillance, Epidemiology, and End Results (SEER) database for the general population has been noted.

In addition, at its May 2011 meeting, the FDA Pediatric Advisory Committee reviewed five observational studies published between January 2005 and April 2011, looking at the outcomes of lymphoma, nonmelanoma skin cancer, melanoma, and other cancers. ${ }^{18}$ In summary, most of the studies reviewed by the FDA committee showed no increased risk for either lymphoma or overall cancer rate. A possible increased risk for T-cell lymphoma was reported with tacrolimus in one study, but the potential confounders discussed included the risks inherent in $\mathrm{AD}$ itself, study biases, and "protopathic bias" (that is, misdiagnosis of AD when the patient actually had cutaneous T-cell lymphoma). The FDA committee concluded that the review of the observational literature suggested a possible association between tacrolimus use and increased risk of T-cell lymphoma, but that there were potential biases to explain this observed association. They also noted that the results were not specific to the pediatric population, that the postmarketing safety review did not identify new signals for pediatric malignancies with the TCIs, and that the current labeling and medication guide reflects the safety risk as currently understood.

A meta-analysis of randomized clinical trials of TCIs for the treatment of $\mathrm{AD}$ in a combined total of 6,288 pediatric patients who met the inclusion criteria showed that both tacrolimus and pimecrolimus were safe and effective in the treatment of $\mathrm{AD}$, and the incidence of adverse events was similar to that seen with vehicle alone. ${ }^{19}$

\section{Approaching the Fearful Parent}

Effective management necessitates the establishment of good rapport with the patient and caregivers, expression of empathy for their concerns and anxieties, frequent follow-up for reinforcement and emotional support, selection of treatment regimens that are complete yet as simple as feasible, and the 


\section{Table Online Resources for Atopic Dermatitis}

Online resources include research organizations/consortia, clinical service providers, patient support networks, and educational resources. Some of these are:

- American Academy of Dermatology (http://www.aad.org/ skin-conditions/dermatology-a-to-z/eczema)

- The Eczema Center (http://www.eczemacenter.org)

- Eczema Support Group (http://www.mdjunction.com/ eczema)

- National Eczema Association (US) (http://www.nationaleczema.org)

- The National Eczema Society (UK) (http://www.eczema.org)

- National Jewish Health Pediatric Atopic Dermatitis Program (http://www.nationaljewish.org/programs/ pediatric/atopic-dermatitis/)

- Talk Eczema (http://www.talkeczema.com)

use of ancillary materials (such as written action plans) to encourage treatment adherence.

Clinicians should address safety and correct use of topical agents directly at the time of their recommendation and anticipate (and answer) questions that parents/caregivers may have. Toward this end, practitioners must be thoroughly educated themselves on the appropriate use and safety profiles of the topical regimens they prescribe.

It is important to address with patients and caregivers the distinction between oral and topical corticosteroids and to dispel the notion that different topical corticosteroid agents can be directly compared to each other based on their percentage strengths (for example, 2.5\% hydrocortisone is not 25 times stronger than $0.1 \%$ mometasone).

Not all topical medications commonly used in infants and young children are specifically indicated by the FDA for patients in these age groups. Clinicians who intend to prescribe use of a medication outside of its approved indications should be up-front with parents about this fact, should explain what "off-label" actually means, and should discuss the fact that off-label therapy is common in treating dermatologic conditions and in pediatric medicine, in general.

The parent education and personal care plan at the end of this article on page S29 was developed by two of the authors (Lawrence F. Eichenfield, MD and Anthony J. Mancini, MD) and is provided as an aid to support clinicians' efforts in reinforcing important information and instructions. It is organized based on the four areas of treatment that must be addressed in patients with AD: dryness, inflammation, itching, and infection. Understanding these four parameters may be useful to parents to help them stay aware of the range of issues that must be addressed in treating their child with AD. (This two-sided handout may be freely duplicated and distributed, without charge, to patients and parents. Other use, such as inclusion in published materials or presentations, requires proper attribution to the authors and permission from the publisher).

\section{Education Beyond the Clinic Visit}

There are many resources beyond the routine clinic visit that may help patients and families understand the process of AD more completely, hence translating into increased rates of adherence. In some cases, referral to online information and other resources can be helpful (see the Table on this page).

Written action plans have been extensively developed and tested in the setting of pediatric asthma and have been shown to help increase adherence with therapy and improve asthma control. They serve as a communication aid between practitioner and patient/parent and also, when kept in a readilyaccessible location in the home, help remind caregivers of the treatment plan. ${ }^{20} \mathrm{~A}$ study on the perceptions of "eczema action plans" for AD found that $100 \%$ of the surveyed pediatricians reported that they would be likely to use an eczemas action plan in their clinical practice. ${ }^{21}$ Although parallel studies are largely lacking in AD, action plans similar to those used in asthma make intuitive sense and are already used by many practitioners, especially pediatric dermatologists. For an action plan to be successful, it should be personalized for the patient and should be clear, concise, and easy to read. Fleischer ${ }^{22}$ noted that written action plans should be quantifiably good and tested.

Various models of delivery of AD education outside of the traditional clinic visit have been developed. These include nurse-led approaches and group instruction (including "eczema school" or weekly educational seminars). ${ }^{23}$ A review of the literature addressing the benefits of nurse-led AD education clinics reviewed 22 relevant publications and supported the efficacy of such clinics in the management of such chronic illnesses. These nurse-led clinics allow for increased time spent with patients, resulting in improved patient education, treatment adherence, and satisfaction with care. ${ }^{24}$

In addition, multidisciplinary clinics for $\mathrm{AD}$ are being formed at many centers. These offer the advantages of comprehensive "one-stop" care, intensive education, focused educational curricula, and access to patient support networks.

\section{Conclusion}

Education is the most important promoter of adherence because action without understanding is unlikely to persist long term. However, true education and understanding can be accomplished only when the provider-patient relationship is effective. Such effectiveness is enhanced when the health provider demonstrates an awareness and understanding of psychosocial issues, preconceived notions, anxieties about treatment safety, and psychological comorbidities relevant to the disease being treated (AD) and takes definitive steps to help patients and parents address them. Other proven promoters of education and adherence include simplified treatment regimens, the prescription of treatments and medications in vehicles preferred by the patient and caregivers, and written action plans and referrals for educational resources and patient support networks. Multidisciplinary clinics for 
$\mathrm{AD}$, where available, simplify the delivery of comprehensive care and intensive patient education.

\section{References}

1. Nolan BV, Feldman SR: Dermatologic medication adherence. Dermatol Clin 27:113-120, 2009

2. Aubert-Wastiaux H, Moret L, Le Rhun A, et al: Topical corticosteroid phobia in atopic dermatitis: A study of its nature, origins and frequency. Br J Dermatol 165:808-814, 2011

3. Richards HL, Fortune DG, O'Sullivan TM: Patients with psoriasis and their compliance with medication. J Am Acad Dermatol 41:581-583, 1999

4. Krejci-Manwaring J, Tusa MG, Carroll C, et al: Stealth monitoring of adherence to topical medication: Adherence is very poor in children with atopic dermatitis. J Am Acad Dermatol 56:211-216, 2007

5. Scholtz JR: Management of atopic dermatitis: A preliminary report. Calif Med 100:103-105, 1964

6. Scholtz JR: Management of atopic dermatitis. Calif Med 102:210-216, 1965

7. Chiang C, Eichenfield LF: Quantitative assessment of combination bathing and moisturizing regimens on skin hydration in atopic dermatitis. Pediatr Dermatol 26:273-278, 2009

8. Gutman AB, Kligman AM, Sciacca J: Soak and smear: A standard technique revisited. Arch Dermatol 141:1556-1559, 2005

9. Niggemann B, Sielaff B, Beyer K: Outcome of double-blind, placebocontrolled food challenge tests in 107 children with atopic dermatitis. Clin Exp Allergy 29:91-96, 1999

10. Sampson HA, Albergo R: Comparison of results of skin tests, RAST, and double-blind, placebo-controlled food challenges in children with atopic dermatitis. J Allergy Clin Immunol 74:26-33, 1984

11. Suh L, Coffin S, Leckerman KH: Methicillin-resistant Staphylococcus aureus colonization in children with atopic dermatitis. Pediatr Dermatol 25:528-534, 2008

12. Krakowski AC, Eichenfield LF, Dohil MA: Management of atopic dermatitis in the pediatric population. Pediatrics 122:812-824, 2008

13. Liu T, Howard RM, Mancini AJ, et al: Kwashiorkor in the United States:
Fad diets, perceived and true milk allergy, and nutritional ignorance. Arch Dermatol 137:630-636, 2001

14. Boyce JA, Assa'ad A, Burks AW, et al: Guidelines for the diagnosis and management of food allergy in the United States: Report of the NIAIDsponsored expert panel. J Allergy Clin Immunol 126:S1-S58, 2010

15. Burks AW, Tang M, Sicherer S, et al: ICON: Food allergy. J Allergy Clin Immunol 129:906-920, 2012

16. Yang LP, Curran MP: Spotlight on topical pimecrolimus in pediatric atopic dermatitis. Am J Clin Dermatol 11:295-298, 2010

17. Ohtsuki M, Ohara H, Santos V: Safety profiles of two large cohort studies of tacrolimus ointment for the treatment of atopic dermatitis: A prospective pediatric longitudinal evaluation study (APPLES) and Japanese long-term safety study (J-LSS). Poster presented at: 22nd World Congress of Dermatology; May 24-29, 2011; Seoul, Korea.

18. Manthripragada A: Topical calcineurin inhibitors and malignancies in pediatric patients: A literature review. Presented at: U.S. Food and Drug Administration Pediatric Advisory Committee Meeting, May 16, 2011. Available at: http://www.fda.gov/downloads/AdvisoryCommittees/ CommitteesMeetingMaterials/PediatricAdvisoryCommittee/UCM255411. pdf. Accessed June 12, 2012.

19. Chen SL, Yan J, Wang FS: Two topical calcineurin inhibitors for the treatment of atopic dermatitis in pediatric patients: A meta-analysis of randomized clinical trials. J Dermatolog Treat 21:144-156, 2010

20. Chisolm SS, Taylor SL, Balkrishnan R: Written action plans: Potential for improving outcomes in children with atopic dermatitis. J Am Acad Dermatol 59:677-683, 2008

21. Ntuen E, Taylor SL, Kinney M, et al: Physicians' perceptions of an eczema action plan for atopic dermatitis. J Dermatolog Treat 21:28-33, 2010

22. Fleischer $A B$ Jr: Written action plans should be comprehensive and evidence-based. J Am Acad Dermatol 65:1059, 2011

23. Nicol NH, Ersser SJ: The role of the nurse educator in managing atopic dermatitis. Immunol Allergy Clin North Am 30:369-383, 2010

24. Moore E, Williams A, Manias E, et al: Nurse-led clinics reduce severity of childhood atopic eczema: A review of the literature. Br J Dermatol $155: 1242-1248,2006$ 